and TACC genes in human glioblastoma. Science. 2012;337(6099):1231-1235

14. Cappellen D, et al. Frequent activating mutations of FGFR3 in human bladder and cervix carcinomas. Nat Genet. 1999;23(1):18-20.

15. Eswarakumar VP, Lax I, Schlessinger J. Cellular signaling by fibroblast growth factor receptors. Cytokine Growth Factor Rev. 2005;16(2):139-149.

16. Fenton TR, et al. Resistance to EGF receptor inhibitors in glioblastoma mediated by phosphorylation of the PTEN tumor suppressor at tyrosine 240. Proc
Natl Acad Sci U S A. 2012;109(35):14164-14169. 17. Gergely F, Kidd D, Jeffers K, Wakefield JG, Raff JW. D-TACC: a novel centrosomal protein required for normal spindle function in the early Drosophila embryo. EMBO J. 2000;19(2):241-252.

18. Still IH, Vince P, Cowell JK. The third member of the transforming acidic coiled coil-containing gene family, TACC 3 , maps in $4 \mathrm{p} 16$, close to translocation breakpoints in multiple myeloma, and is upregulated in various cancer cell lines. Genomics. 1999;58(2):165-170.
19. Fabian MR, Sonenberg N, Filipowicz W. Regulation of mRNA translation and stability by microRNAs. Annu Rev Biochem. 2010;79:351-379.

20. Mayr C, Bartel DP. Widespread shortening of 3'UTRs by alternative cleavage and polyadenylation activates oncogenes in cancer cells. Cell. 2009; 138(4):673-684.

21. Sandberg R, Neilson JR, Sarma A, Sharp PA, Burge CB. Proliferating cells express mRNAs with shortened 3' untranslated regions and fewer microRNA target sites. Science. 2008;320(5883):1643-1647.

\title{
Old King Coal - molecular mechanisms underlying an ancient treatment for atopic eczema
}

\author{
W.H. Irwin McLean' and Alan D. Irvine ${ }^{2,3,4}$
}

\begin{abstract}
${ }^{1}$ Centre for Dermatology and Genetic Medicine, Division of Molecular Medicine, Colleges of Life Sciences and Medicine, Dentistry and Nursing, University of Dundee, Dundee, United Kingdom. '2Department of Paediatric Dermatology, Our Lady's Children's Hospital, Dublin, United Kingdom. ${ }^{3}$ Clinical Medicine, Trinity College Dublin, Dublin, United Kingdom. ${ }^{4}$ National Children's Research Centre,

Our Lady's Children's Hospital, Dublin, United Kingdom.
\end{abstract}

\begin{abstract}
Traditional remedies for common disorders have been known for centuries, but insight into their mechanism of action is often limited. In this issue of the JCI, Joost Schalkwijk's research group at the Radboud University Nijmegen Medical Centre in The Netherlands advances our understanding of why topical coal tar is an effective treatment for atopic dermatitis (AD), both rationalizing the use of this traditional medicine, and providing the scientific basis for new therapeutic approaches.
\end{abstract}

$\mathrm{AD}$ (also called "eczema") is the most common inflammatory skin condition, affecting about $20 \%$ of children in the developed world. AD is a classic complex trait where a combination of several genetic predisposing factors interact with environmental stimuli to trigger the disease. AD is frequently associated with high serum $\operatorname{IgE}$ and a Th2 immune response $(1,2)$. In 2006, a paradigm shift in the pathomechanistic understanding of $\mathrm{AD}$ took place when loss-of-function mutations were discovered in the FLG gene encoding the skin barrier protein filaggrin in the common monogenic skin disease ichthyosis vulgaris (dry, flaky skin) (3). Soon thereafter, these same filaggrin variants, which are carried by about $10 \%$ of populations of European ancestry and persist at high frequencies in other populations (4), were shown to be the major genetic predisposing factor in $\mathrm{AD}$ (5). Filaggrin-deficient animals were subsequently shown to have a "leaky" skin bar-

Conflict of interest: The authors have declared that no conflict of interest exists.

Citation for this article: J Clin Invest. 2013; 123(2):551-553. doi:10.1172/JCI67438. rier, allowing passive percutaneous transfer of antigens, which trigger skin inflammation and an allergic immune response, analogous to AD in humans (6). This work showed that a primary skin barrier deficiency is at least one important factor in $\mathrm{AD}$ pathogenesis, although Th2 immunity is clearly also a major player (1).

\section{Making the pitch for pitch}

Coal tar has been used medically since ancient times. In his epic 5-volume work,

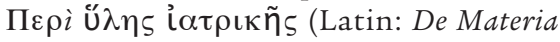
Medica), the Greek physician, pharmacologist, and botanist Pedanius Dioscorides (circa 40-90 CE) chronicled many and varied herbal and other remedies in use at the time, including the "grime of a gymnasium wall." While this remedy for abrasions and ulcers does not currently enjoy popularity, his suggestion of the use of bitumen or asphalt/coal tar for "inflammation" (7) has maintained traction over the succeeding 2 millennia (8). For as long as modern dermatology departments have existed, liquor picis carbonis (LPC) has been a part of their working vocabulary, and preparations containing LPC are widely considered to be effective in the treatment of psoriasis and AD. Indeed, a recent systematic review provided evidence of the efficacy of $0.5 \%-5 \%$ LPC preparations for both these conditions (9).

Tar, asphalt, bitumen, and pitch are related substances consisting of complex mixtures of high molecular weight organic compounds that can be derived from heat distillation of plants, wood, petrochemicals, or coal. Pitch essentially functions as a solid but is really an incredibly viscous liquid that is estimated to have more than 100 billion times the viscosity of water (10). The world's longest continuously running laboratory experiment is The University of Queensland's "Pitch Drop Experiment" (10). Begun in 1927-1930 by Thomas Parnell (it took 3 years just for the pitch to settle into a glass funnel), droplets of pitch fall under gravity only about once a decade, with the next one expected this year. We live in exciting times!

\section{A target identified}

Although the terms are somewhat ambiguous and interchangeable, pitch tends to refer to the more solid substances in this group, and tar generally refers to the more liquefied products. Coal tar is an extremely viscous liquid obtained from dry-heating coal to temperatures in the range of $900^{\circ} \mathrm{C}$ to $1200^{\circ} \mathrm{C}$, and is thought to consist of at least 10,000 distinct high molecular weight hydrocarbon and aromatic compounds, 
A

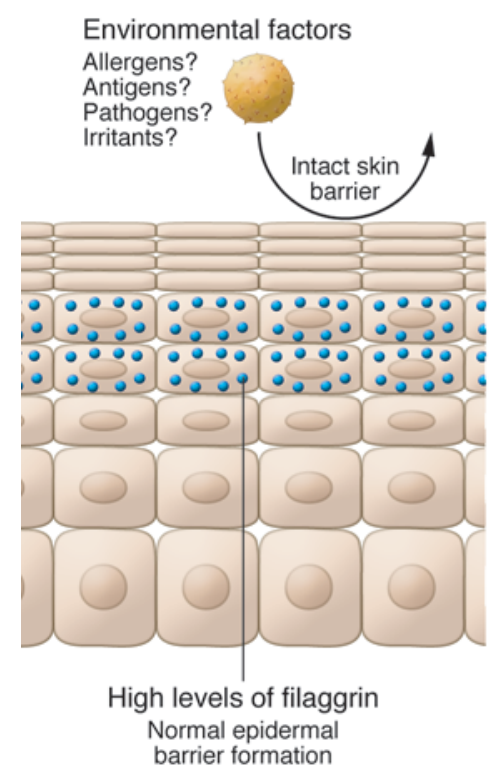

B

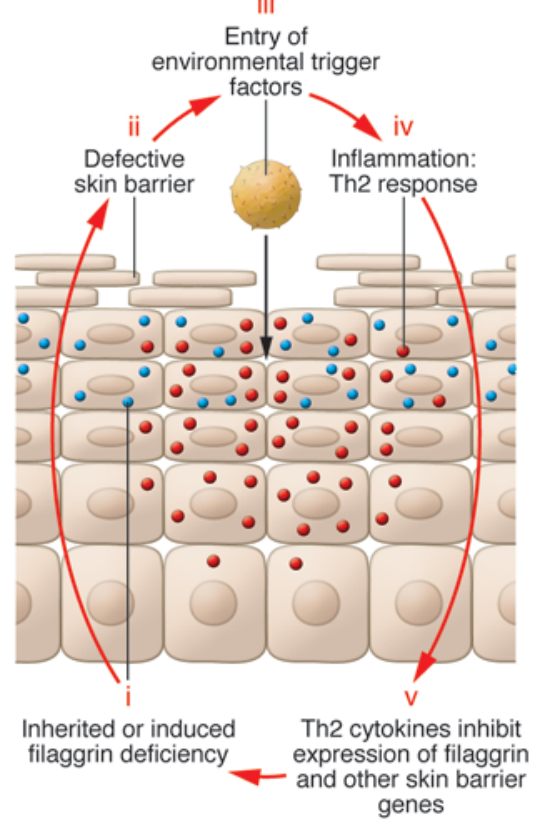

C

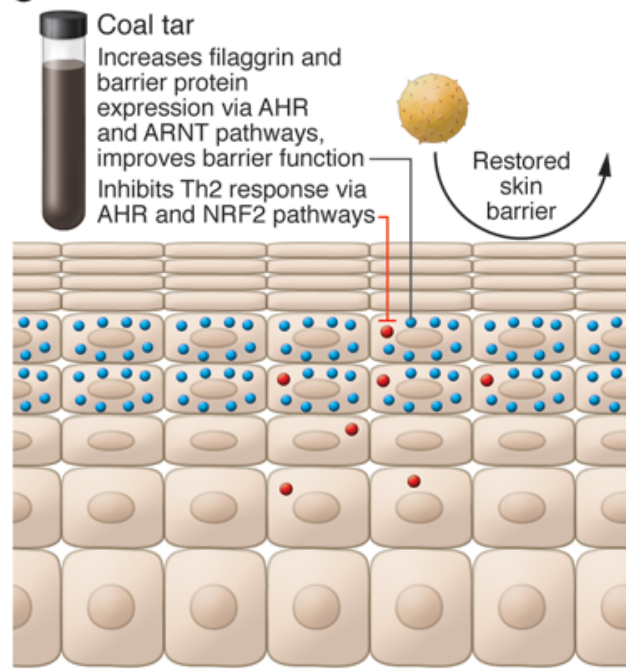

\section{Figure 1}

Proposed mechanism of action of coal tar in AD. (A) Schematic of the epidermis showing the expression of filaggrin (and related proteins involved in the biogenesis of the skin barrier) within the granular cell layers that underlie the dead, terminally differentiated squames of the stratum corneum where the skin's barrier function mainly resides. An intact skin barrier prevents water loss as well as entry of foreign material into the body. (B) In many patients with $A D$, there is either an inherited defect in barrier formation (e.g., due to filaggrin deficiency) and/or indirect repression of skin barrier genes (e.g., by the action of Th2 cytokines). The defective barrier allows entry of foreign substances, which leads to an inflammatory response where Th2 cytokines are especially important. These cytokines further repress skin barrier formation, thereby completing a negative feedback loop. (C) Schalkwijk's group show here that coal tar acts via the AHR, which has two beneficial effects: (a) increasing epidermal differentiation and upregulating the expression of key barrier proteins such as filaggrin, and (b) suppressing the Th2 cytokine response. Thus, coal tar is able to break the mechanistic cycle in AD.

depending on the type of coal used (11). Coal tar is particularly rich in polycyclic aromatic hydrocarbons (PAHs), which most likely account for the link to epidermal biology, as demonstrated by van den Bogaard et al. in this issue (12).

Epidermal keratinocytes internalize PAHs and detoxify them using the cytochrome P450 family of enzymes, more than a dozen of which are highly expressed in the epidermis (13). Although the liver remains the primary detoxification organ, the role of the epidermis in the metabolism of drugs and other xenobiotic chemicals is increasingly recognized (14). Van den Bogaard et al. show that the effects of coal tar are mediated through aryl hydrocarbon receptor (AHR) activation. AHR activation has the highly desirable and doubly beneficial effect of both directly enhancing epidermal differentiation through restoration of key epidermal barrier proteins and blocking the effects of Th2 cytokines, thus removing a secondary repressor of skin barrier function (Figure 1).

\section{Clean coal?}

It has recently been shown that coal tar applied to the skin is absorbed at low levels and excreted in the urine, making both systemic immunomodulatory effects and systemic carcinogenic effects possible (15). Given that AD most often initially presents in children less than 2 years of age, when transcutaneous absorption is enhanced and drug metabolic pathways may be immature and less effective, a proven safety profile would be very desirable. To this end, identification and purification of the beneficial pharmacologically active moieties in coal tar from those that are potentially carcinogenic would be of great interest for future drug development. There are two obvious approaches to refine coal tar therapy based on the data presented here by the Schalkwijk group. Chromatographic fractionation of coal tar could be employed to identify the key active components therein that activate the AHR, however, it is likely that a large percentage of the PAHs within this complex mixture will target AHR and few, if any, are likely to have the desirable drug-like chemical properties upon which to build a drug discovery program. Alternatively, high-throughput screening of chemical compound libraries could be used to identify pharmacologically tractable small molecules that act through the AHR and other downstream pathways.

While the effects of a complex organic compound mixture such as coal tar are likely to be pleiotropic, these new insights identify the AHR as a key regulatory pathway and therefore a potential drug target for AD. Although the differential effects of dioxin versus coal tar exposure on the AHR will likely need to be resolved by further experimental work in order to convince regulatory authorities to reassess the viability and safety of potential drugs that act on this pathway, the work of van den Bogaard et al. nevertheless sets the scene for an exciting new rational drug design program in this important common disease. 


\section{Acknowledgments}

The authors' research into the role of filaggrin in the genetics of atopic eczema is funded by Wellcome Trust Programme (092530/Z/10/Z; to W.H.I. McLean and A.D. Irvine), and Bioresources grants (090066/B/09/Z; to A.D. Irvine and W.H.I. McLean). The Centre for Dermatology and Genetic Medicine, University of Dundee is supported by a Wellcome Trust Strategic Award (098439/Z/12/Z; to W.H.I. McLean).

Address correspondence to: W.H. Irwin McLean, Centre for Dermatology and Genetic Medicine, Division of Molecular Medicine, Colleges of Life Sciences and Medicine, Dentistry and Nursing, University of Dundee, Dow Street, Dundee DD1 5EH, United Kingdom. Phone: 44.1382.381046; Fax: 44.1382.388535; E-mail: w.h.i.mclean@dundee.ac.uk.
1. Irvine AD, McLean WHI, Leung DY. Filaggrin mutations associated with skin and allergic diseases. N Engl J Med. 2011;365(14):1315-1327.

2. Novak N, Leung DY. Advances in atopic dermatitis. Curr Opin Immunol. 2011;23(6):778-783.

3. Smith FJD, et al. Loss-of-function mutations in the gene encoding filaggrin cause ichthyosis vulgaris. Nat Genet. 2006;38(3):337-342.

4. Sandilands A, et al. Comprehensive analysis of the gene encoding filaggrin uncovers prevalent and rare mutations in ichthyosis vulgaris and atopic eczema. Nat Genet. 2007;39(5):650-654.

5. Palmer CNA, et al. Common loss-of-function variants of the epidermal barrier protein filaggrin are a major predisposing factor for atopic dermatitis. Nat Genet. 2006;38(4):441-446.

6. Fallon PG, et al. A homozygous frameshift mutation in the mouse Flg gene facilitates enhanced percutaneous allergen priming. Nat Genet. 2009;41(5):602-608.

7. Dioscorides P. Book 1: Aromatics. In: Osbaldeston TA, Wood RPA, eds. Dioscorides: De Materia Medica (A new indexed version in modern English). Johannesburg, South Africa: Ibidis Press; 2000:99-100.

8. Thami GP, Sarkar R. Coal tar: past, present and future. Clin Exp Dermatol. 2002;27(2):99-103

9. Slutsky JB, Clark RA, Remedios AA, Klein PA.
An evidence-based review of the efficacy of coal tar preparations in the treatment of psoriasis and atopic dermatitis. J Drugs Dermatol. 2010; 9(10):1258-1264.

10. Mainstone J. The Pitch Drop Experiment. The University of Queensland Web site. http://smp.uq.edu. $\mathrm{au} /$ content/pitch-drop-experiment. Accessed December 10, 2012

11. Choudhary G, Citra MJ, McDonald AR, QuiñoneRivera A. Toxicological Profile for Wood Creosote, Coal Tar Creosote, Coal Tar, Coal Tar Pitch, and Coal Tar Pitch Volatiles. Atlanta, Georgia, USA; US Department of Health and Human Services; 2002.

12. van den Bogaard EH, et al. Coal tar induces AHRdependent skin barrier repair in atopic dermatitis. J Clin Invest. 2013;123(2):917-927.

13. Du L, Hoffman SM, Keeney DS. Epidermal CYP2 family cytochromes P450. Toxicol Appl Pharmacol. 2004;195(3):278-287.

14. Smith G, et al. Regulation of cutaneous drugmetabolizing enzymes and cytoprotective gene expression by topical drugs in human skin in vivo. Br J Dermatol. 2006;155(2):275-281.

15. Roelofzen JH, et al. DNA adducts in skin biopsies and 1-hydroxypyrene in urine of psoriasis patients and healthy volunteers following treatment with coal tar. Toxicol Lett. 2012;213(1):39-44.

\title{
Nucleocytoplasmic connections and deafness
}

\author{
Howard J. Worman ${ }^{1}$ and Neil Segil2,3
}

1Departments of Medicine and of Pathology and Cell Biology, College of Physicians and Surgeons, Columbia University, New York, New York, USA. 2Division of Cell Biology and Genetics, House Research Institute and ${ }^{3}$ Department of Cell and Neurobiology, Keck School of Medicine, University of Southern California, Los Angeles, California, USA.

\begin{abstract}
The linker of nucleoskeleton and cytoskeleton (LINC) complex connects the nuclear lamina to the cytoskeleton, in part to aid in nuclear positioning. Mutations in genes encoding LINC complex and lamina components cause a range of human diseases. In this issue of the JCI, Horn et al. report that mutations in the gene SYNE4 encoding the LINC complex protein nesprin-4 lead to progressive high-frequency hearing loss. Further, in mice deficient in nesprin-4 and Sun1, another LINC complex component, outer hair cells of the cochlea form normally during development, but die in the early postnatal weeks. These results link improper nuclear positioning specifically to the death of outer hair cells in the organ of Corti and ultimately to deafness.
\end{abstract}

The nuclear envelope is composed of the nuclear membranes (inner and outer), nuclear pore complexes, and nuclear lamina. It separates the nucleoplasm from the cytoplasm of eukaryotic cells, and the transport of proteins, nucleic acids, and other molecules among these compartments in interphase is restricted to the pore complexes. The nuclear envelope has been a growing focus of

Conflict of interest: The authors have declared that no conflict of interest exists.

Citation for this article: J Clin Invest. 2013; 123(2):553-555. doi:10.1172/JCI67454. clinical investigation, as in the past 15 years a wide range of inherited diseases have been linked to mutations in genes encoding proteins of this subcellular structure $(1,2)$.

\section{Nuclear envelope and nucleocytoplasmic connections}

Recent research has shown that the nuclear envelope not only separates the nucleus from the cytoplasm, but also connects the structural networks of these subcellular compartments (Figure 1). The linker of nucleoskeleton and cytoskeleton (LINC) complex mediates this con- nection (3). The core of the LINC complex forms from the interaction of SUN (Sad1, UNC-84) domain proteins with KASH (Klarsicht, ANC-1, Syne homology) domain proteins called nesprins (4, 5 ). This creates a protein bridge spanning the inner and outer nuclear membranes. The SUN domains, attached to a trimeric coiled coil of Sun proteins, bind three KASH domains, with a disulfide bond between cysteines in SUN and KASH further covalently linking them (6). Suns are integral proteins of the inner nuclear membrane that also bind to A-type lamins of the nuclear lamina, a meshwork of intermediate filament proteins providing structural support to the nucleus. The SUN-KASH binding within the perinuclear space retains the nesprin, which also contains a transmembrane segment, in the outer nuclear membrane. At the other end of this transnuclear membrane bridge, different nesprins interact with unique cytoskeletal components. For example, nesprin-1 and nesprin-2 isoforms bind directly to actin, nesprin-3 via plectin to cytoplasmic intermediate 